\title{
Research on Monitoring System of Building Slope Deformation Based on Internet of Things
}

\author{
Yang Xinfa ${ }^{1}$, Liu Qiong ${ }^{2, *}$ \\ ${ }^{1}$ Hainan Institute of Marine Geological Survey, Haikou, Hainan Province, China \\ ${ }^{2}$ Hainan Hydrogeological Engineering Geological Survey Institute, Haikou, Hainan Province, China
}

\begin{abstract}
A building slope deformation monitoring system based on the Internet of Things technology was studied in this paper. Through the powerful data acquisition, storage, calculation and analysis capabilities of new technologies such as sensors, cloud computing, big data communication and visualization, and a variety of monitoring data acquisition instruments, sensors, communication terminals, cloud platform and other software and hardware resources as the carrier, it can realize real-time online equipment, continuous data acquisition, real-time calculation, data analysis, emergency warning, and the whole process of deformation monitoring services. Our research solves the deficiency of traditional technical means and effectively improves the timeliness, reliability and information level of construction slope deformation monitoring.
\end{abstract}

\section{Introduction}

The building slope refers to the artificial slope formed by the excavation or filling construction of the construction project and the municipal engineering, and the natural slope that adversely affects the safety or stability of the building (structure) on the building site and its surroundings. [1] According to the requirements of "Technical Code for Building Slope Engineering" (GB 50330) and "Technical Code for Appraisal and Reinforcement of Building Slope Engineering" (GB 50843), during the construction of the slope, the horizontal displacement, vertical displacement, surface crack and deformation of the slope roof must be monitored. The monitoring should meet the requirements of information method construction and timely providing monitoring data and reports. ${ }^{2]}$

Traditional deformation monitoring methods rely on manual operation of total station, level, stress meter, inclinometer, water level gauge and other instruments and equipment to obtain deformation data at each monitoring point of the building slope during the construction and use of building slopes. The large amount of monitoring data and diverse types lead to low processing efficiency; single calculation and analysis methods make it difficult to guide on-site dynamic design, construction and optimization of design parameters; result data sharing and early warning methods are low in information, especially in the case of serious deformation of the construction slope, the requirements of timeliness of construction and operation safety will not be satisfied. Therefore, based on the new technology of the Internet of Things, a building slope deformation monitoring system is researched to realize real-time, continuous and long-term monitoring, calculation and analysis of various types of monitoring points, and then to develop, implement and optimize design scheme and adjust design parameters to provide accurate, reliable, timely technical basis, and master the deformation characteristics and rules of the construction slope at each stage of construction and utilization, which have important social and economic values.

This paper was based on the new technology of the Internet of Things, combined with the construction slope deformation monitoring work carried out by the author, various types of sensors such as deformation, force change, water level and communication application technology was used to study a building slope deformation monitoring system. This system can satisfy the overall requirements of rapid response, continuous improvement, friendly interface, powerful functions, and easy operation. Based on the frequency requirements of the specification and design, the change value of various physical quantities of the monitored object can be measured, collected, and processed, the data can be transmitted through a wired or wireless network, and data analysis is conducted and released by the data management central control platform.

\section{Introduction to the Internet of Things}

Internet of Things (Internet of Things, referred to as IOT) is a kind of network refers to radio frequency identification (RFID; which is a communication technology that can identify a specific target by radio signals and the reading and writing of relevant data without the need to establish mechanical or optical

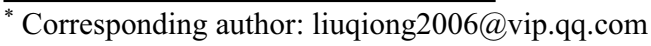


contact between the recognition system and a specific target), infrared (infrared, which is a kind of electromagnetic wave) sensor, global positioning system, laser (Light Amplification by Stimulated Emission of Radiation, LASER; Radiated light derived from stimulated atom, hence the name "laser") scanners and other information sensing equipment, , connect any item to the Internet for information exchange and communication to achieve intelligent identification, positioning, tracking, monitoring and management according to the agreed protocol. ${ }^{[3]}$

From the definition of the Internet of Things, it can be seen that the core and foundation of the Internet of Things is still the Internet, but it has three distinct characteristics and structures: (1) Perception layer, a variety of sensors, multi-source, comprehensive and accurate sources for collecting object information, with comprehensive perceived characteristics; (2) The network layer, public networks, private networks, and cloud computing platforms can quickly process massive amounts of information and have the characteristics of reliable transmission; (3) Application layer, which extracts information and integrates intelligent applications for different industries, with intelligence processing characteristics.

Building slope deformation monitoring is a long-term and continuous work. The construction of a deformation monitoring system centered on the Internet of Things has significant advantages over traditional technical methods: (1) Remote real-time data collection, reducing on-site work costs, reducing personnel and instrument safety hazards; (2) The timeliness and reliability of data collection are greatly improved, and man-made collection errors are reduced; (3) The reliability of information analysis and processing is high, and it can be distributed in real time by SMS, WeChat, email and other methods, and feedback is rapid. In short, this technology can improve the collection and processing efficiency and quality of results of deformation monitoring, especially when the deformation is abnormal, it can realize real-time continuous data collection, calculation processing, and information distribution, and play a more active role in analysis and decision-making.

\section{Technical route}

Based on the full investigation of building slope deformation monitoring technology, data processing methods and data processing system progress, this paper has clarified the problems that the system should cover because of the lack of practicability and timeliness in the current deformation monitoring work that cannot meet the needs of engineering practice. The work content is to carry out research from the two aspects of hardware development and software design, and carry out the overall planning of the deformation monitoring system according to the perception layer, network layer, and application layer, so that the system can meet the technical requirements of building slope deformation monitoring, software development design rules and software Quality of ease of use, effectiveness and reliability. The technical route is shown in Figure 1.

To construct the deformation monitoring system of the Internet of Things, the urgent requirements that need to be met include: (1) The hardware has sufficient scale and capacity to remotely process the data information collected by various sensor terminals on site; (2) The communication integration function is complete, supporting multiple sensor interfaces and data transmission; (3) The database format should be unified, standard, and standardized, and meet the requirements of relevant national and industry standards such as the "Computer Software Documentation Specification" (GB/T 8567) database design standard.

\section{Architecture and function design}

\subsection{System Architecture}

The construction of the building slope deformation monitoring system is designed according to the threelayer structure of the perception layer, network layer and application layer of the Internet of Things, and adopts an easy-to-expandable modular design method, mainly including: (1) The perception layer, which is the core part of Internet of Things, is responsible for data perception and collection, mainly including various sensors (such as water level gauges, strain gauges, displacement gauges, inclinometers, etc.), video monitoring equipment and transmission intermediaries that are responsible for collecting data installed on site. (2) The network layer, including various wired and wireless network communication hardware and software, is responsible for receiving and transmitting various data information collected on site, and providing services to the application layer. (3) The application layer is the core part of the connection between the Internet of Things and users. It mainly uses the data collected on site for analysis and processing, project management, results display, early warning and feedback and other intelligent applications. The overall structure is shown in Figure 2.

\subsection{System core function modules}

According to the overall design idea of the three-tier architecture of the Internet of Things, the system mainly has three core function modules, namely, terminal collection function module, data communication function module, and application service function module. The terminal collection function module mainly collects basic deformation monitoring data through GNSS equipment, strain gauges, displacement gauges, water level gauges and various sensor terminal devices; the data communication function module mainly collects various deformation raw data collected by terminal sensors through various real-time transmission of communication technology, such as $4 \mathrm{G} / 5 \mathrm{G}$, Wi-Fi, LORA, ZigBee and other communication technology means; application service function module is a port connecting the system and users, receiving, storing, 
calculating and analyzing various monitoring data information in real time, and distributing the processed communication protocol, etc. After the data collection is completed, it is transmitted to the management background module in real time and the data quality is

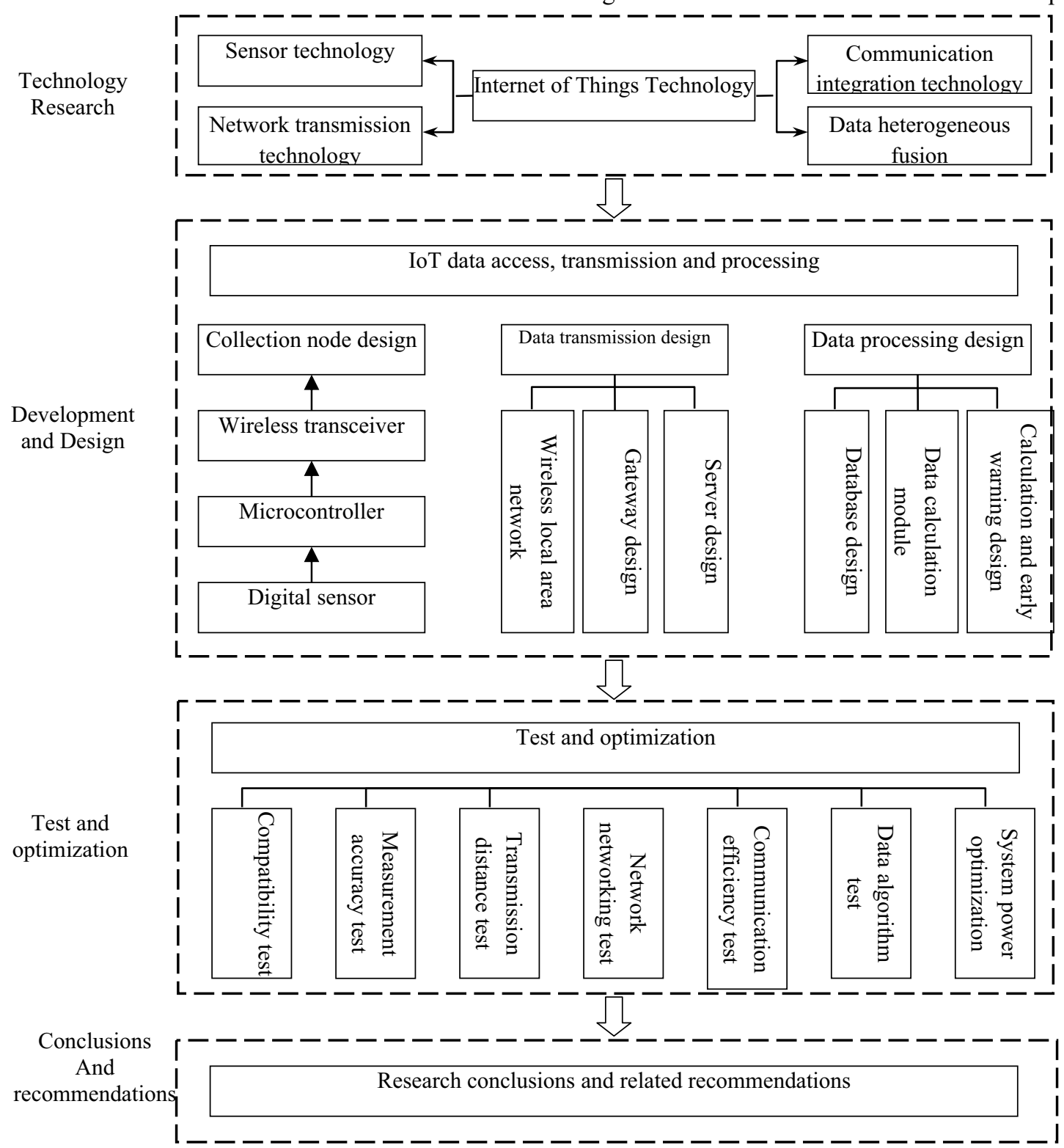

Fig. 1. Technology roadmap.

data for feedback, and providing multi-channel early warning services through SMS, WeChat, and email.

\subsubsection{Terminal collection function module}

The terminal acquisition module is the front end of the entire system. Due to the different protocols and communication modes of different data acquisition equipment, a layered structure modular design method is adopted to realize the communication function between the hardware of each module and the background. After the hardware equipment (various types of monitoring sensors) is constructed and installed according to the needs of each monitoring project, the system management background module sends remote collection instructions through the network. The instructions include collection start time, collection frequency, collection duration, collection end time, evaluated. The qualified data is standardized and saved in the database in a standardized format, and the unqualified data is immediately sent an instruction to recollect.

The main functions of this functional module include monitoring sensor addition, deletion, update management; connection and disconnection status monitoring management; monitoring data remote upload, download, acquisition management, etc.

\subsubsection{Data communication function module}

The data communication function module includes two important parts: one is the data transmission network between the multi-type sensor equipment established by the perception layer and the terminal collection function module, and the appropriate transmission method is selected according to the actual situation of the scene, 
such as RS232 serial port, Bluetooth and Wi -Fi and other transmission methods; the other one is the data transmission network between the terminal collection function module and the background application service function module. Considering the actual needs such as long transmission distance, high data transmission through the MySQL database engine, and complete the addition, deletion, update, and export of various monitoring data information and other attribute information management of attribute data and spatial data, and conduct personalized, refined and systematic inspection and correction.

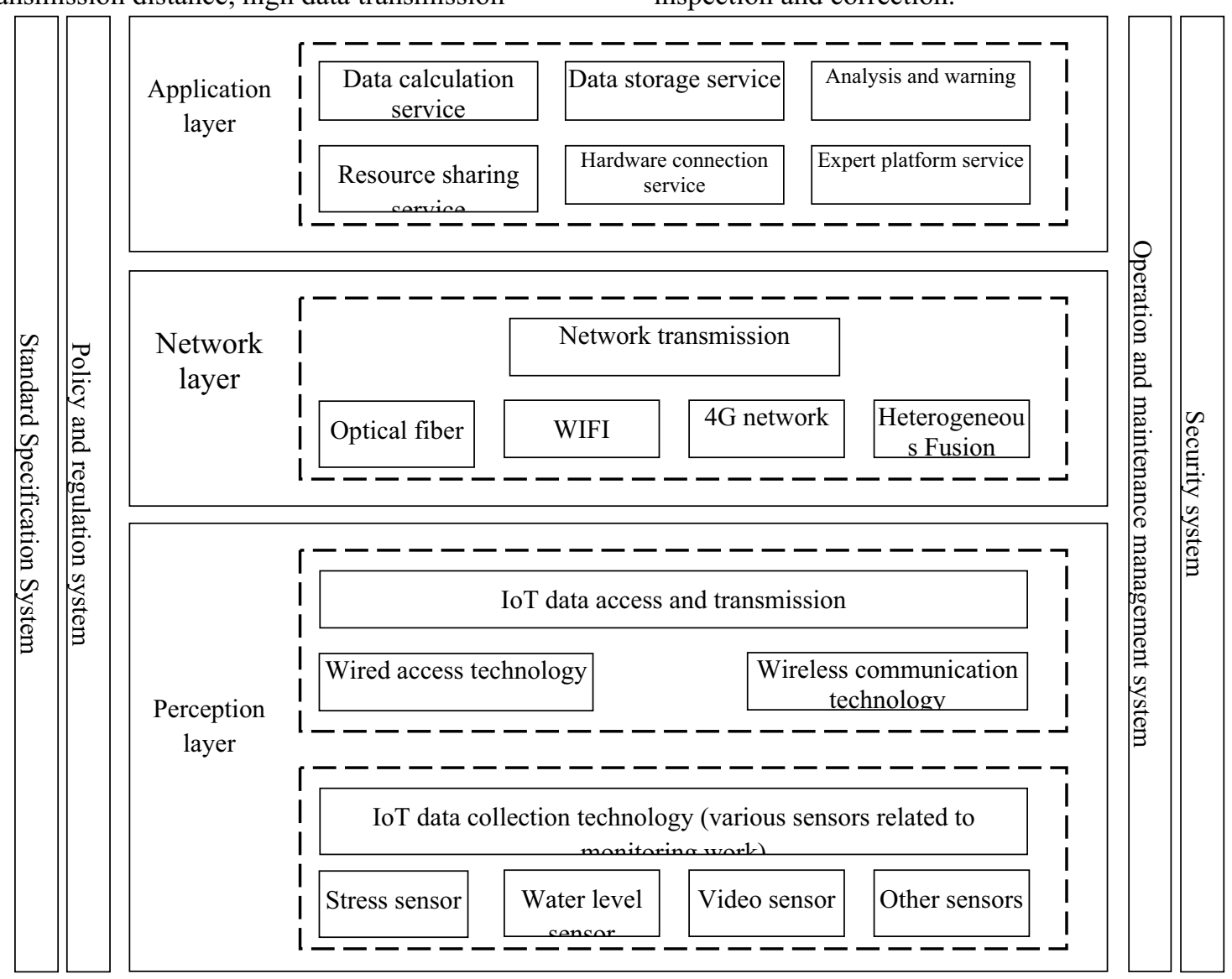

Fig. 2. Overall architecture diagram.

stability requirements, and large transmission data volume, it is generally selected $4 \mathrm{G}$, WIFI and other communication transmission methods.

The main workflow of this functional module is to first collect data from each sensor collection device, and then transmit it to the smart terminal collection device (DTU) in a unified format according to the standard format. The smart terminal collection device compresses and packs the data and transmits it to the cloud platform for supply. The service function module performs calculation, analysis, early warning and feedback, etc.

\subsubsection{Application service function module}

Because building slope monitoring generally has a long working period and various monitoring contents, the application service function module should fully consider the characteristics of large amount of monitoring data, multiple types of data formats, and different forms of data results feedback. Application services should ensure be stable and reliable during design. Using the Internet cloud platform can realize the real-time processing, storage, analysis, early warning, and distribution of deformation monitoring data, interactively access various types of monitoring data
The data sharing and distribution sub-module can be set according to the authority, and automatically or semiautomatically release various types of information to the government administrative supervision department, project construction unit, design unit, surrounding residents, etc., including various monitoring data results, analysis results, early warning and forecasting, risk research and judgment, etc., to provide important information for design, construction, safety assurance, etc., and to play an important reference value.

\section{Summary and Outlook}

This paper is based on the Internet of Things technology, comprehensively using today's rapidly developing sensor technology, cloud platform, wireless networking and communication technology to construct a building slope deformation monitoring system to realize real-time online equipment, continuous data acquisition, real-time calculation, data and the whole-process deformation monitoring service of analysis and emergency warning has effectively improved the response speed, accuracy and reliability of building slope deformation monitoring. It has been successfully applied in some projects and obtained the software copyright registration certificate 
(registered) issued by the National Copyright Administration. No.: 2020SR0735421). In the future, it will further strengthen research on building slope stability analysis, three-dimensional modeling, BIM application, etc., to improve the reliability, accuracy and standardization level of deformation monitoring work, and accelerate the Internet of Things technology in the field of building slope deformation monitoring promotion and application.

\section{Acknowledgments}

This project is funded by High-Level Talent Project of Hainan Natural Science Foundation (SQ2019MSXM0462) and Provincial State-owned Capital Operation Project of Hainan Provincial Department of Finance in 2020(2020-01).

\section{References}

1. National Standards of the People's Republic of China. (2013) Technical code for building slope engineering, China Architecture \& Building Press, Beijing.

2. National Standards of the People's Republic of China. (2013) Technical code for appraisal and Reinforcement of building Slope, China Architecture \& Building Press, Beijing.

3. Jin, Y. Gong, S. (2019) Automatic monitoring technology and practice of slope and foundation pit, Geological Publishing House, Beijing. pp. 37-38.

4. Tang, S. (2017) Php web programming and Ajax technology, Tsinghua university press, Beijing. pp. 254-322.

5. Li, Q. Mei, Y. Meng, S. Zhang, Q. (2016)Design and application of automatic deformation monitoring system based on Internet plus, Bulletin of Surveying and Mapping. pp. 146-147.

6. Lin, D. (2014)Design and implementation of early warning information system for mountain torrents in Yueyang City. Hunan University, Hunan.

7. Xiao, F. (2014)Research on geological informatization based on Internet of things and cloud computing in big data Era, Electronic Technology \& Software Engineering. pp. 221.

8. Zhao, G. (2013)Big data technology and Application Practice Guide, Electronic Industry Press, Beijing. pp. 55-56.

9. Yao, Q. (2018)Geotechnical engineering automatic monitoring system and its application analysis, Building Technology Development. 45(13): 76-77. 